\title{
Severe reaction to inadvertent intravenous administration of norepinephrine
}

\author{
C. Monteiro ${ }^{1}$, S. Ribeiro ${ }^{1}$, A. Gouveia ${ }^{1}$, A. Lares ${ }^{1}$ \\ ${ }^{1}$ Centro Hospitalar Universitário do Algarve - Unidade de Faro - Faro (Portugal)
}

\section{Background:}

Norepinephrine (NE) is an $\alpha 1$ and $\beta 1$ agonist, used for its arterial, venous vasoconstrictor, and positive inotropic properties.[1] However, accidental intravenous (IV) NE is known to result in serious cardiovascular side effects which may include hypertension (HT), angina, arrhythmia, acute myocardial infarction (AMI) and ventricular fibrillation with cardiac arrest. $[2,3]$

Case Report:

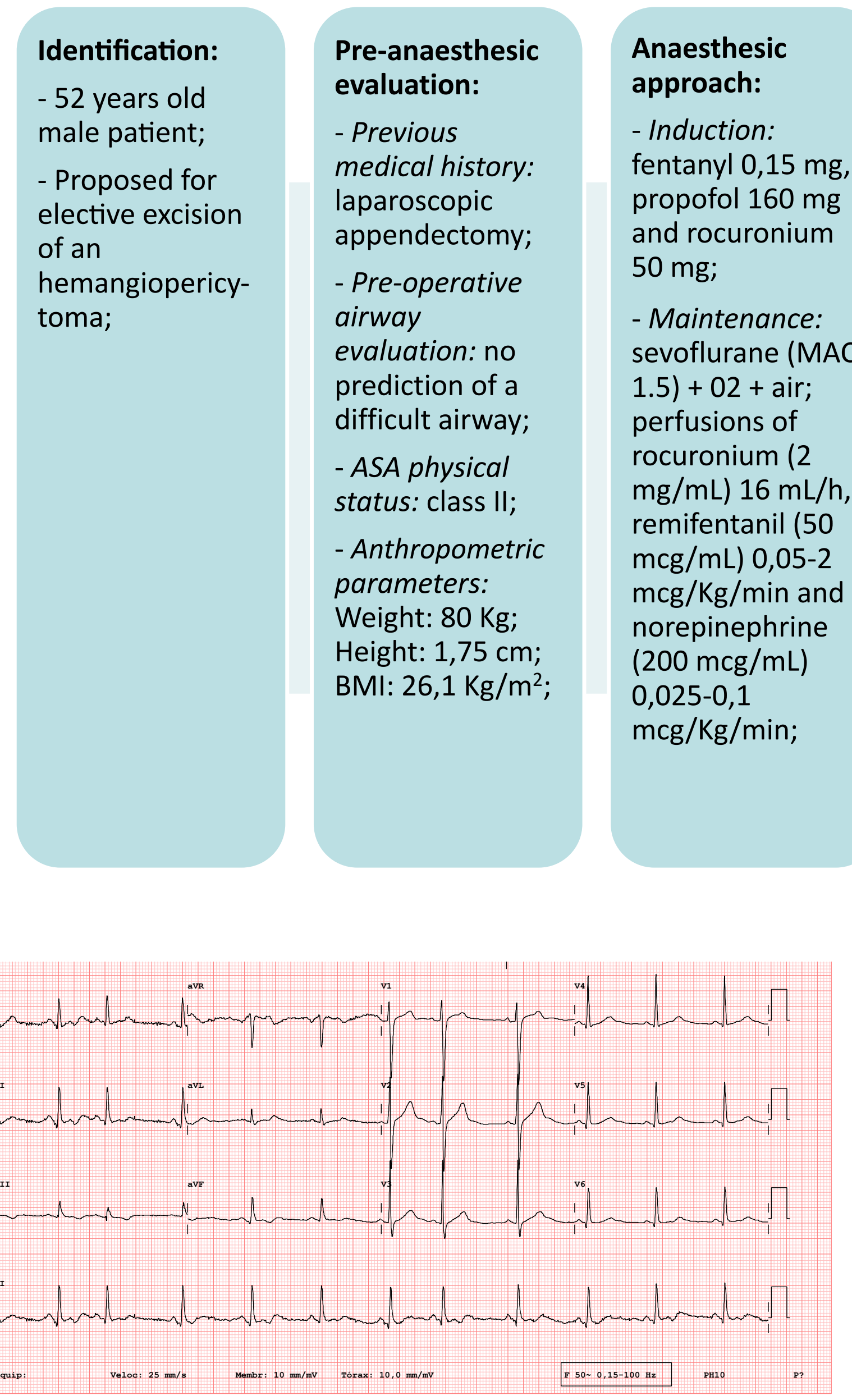

Critical event:
- Upon final
stages of the
surgery, as
perfusions were
stopped, an
accidental
administration of
1 mg of NE
occured;
- Shortly
thereafter, the
patient
developed
supraventricular
tachycardia up to
190 bpm and
arterial HT
(205/110 mmHg);

Critical event:
- ECG showed
signs of
myocardial
ischemia with ST-
segment
elevation in the
D2 and V5 leads.
Afterwards,
arterial pressure
dropped abruptly
to $80 / 40$ mmHg;
- After
hemodinamic
stabilitation, the
surgery was
finished and the
patient was then
admitted in the
ICU;

Recovery:
- ECG showed no
signs compatible
with ischemia nor
AMI;
- Cardiac
biomarkers were
within normal
range;
- No signs of
congestion were
identified in chest
X-ray;
- Patient was
then extubated
uneventfully;
- Discharge in
stable condition
occurred past 6
days.

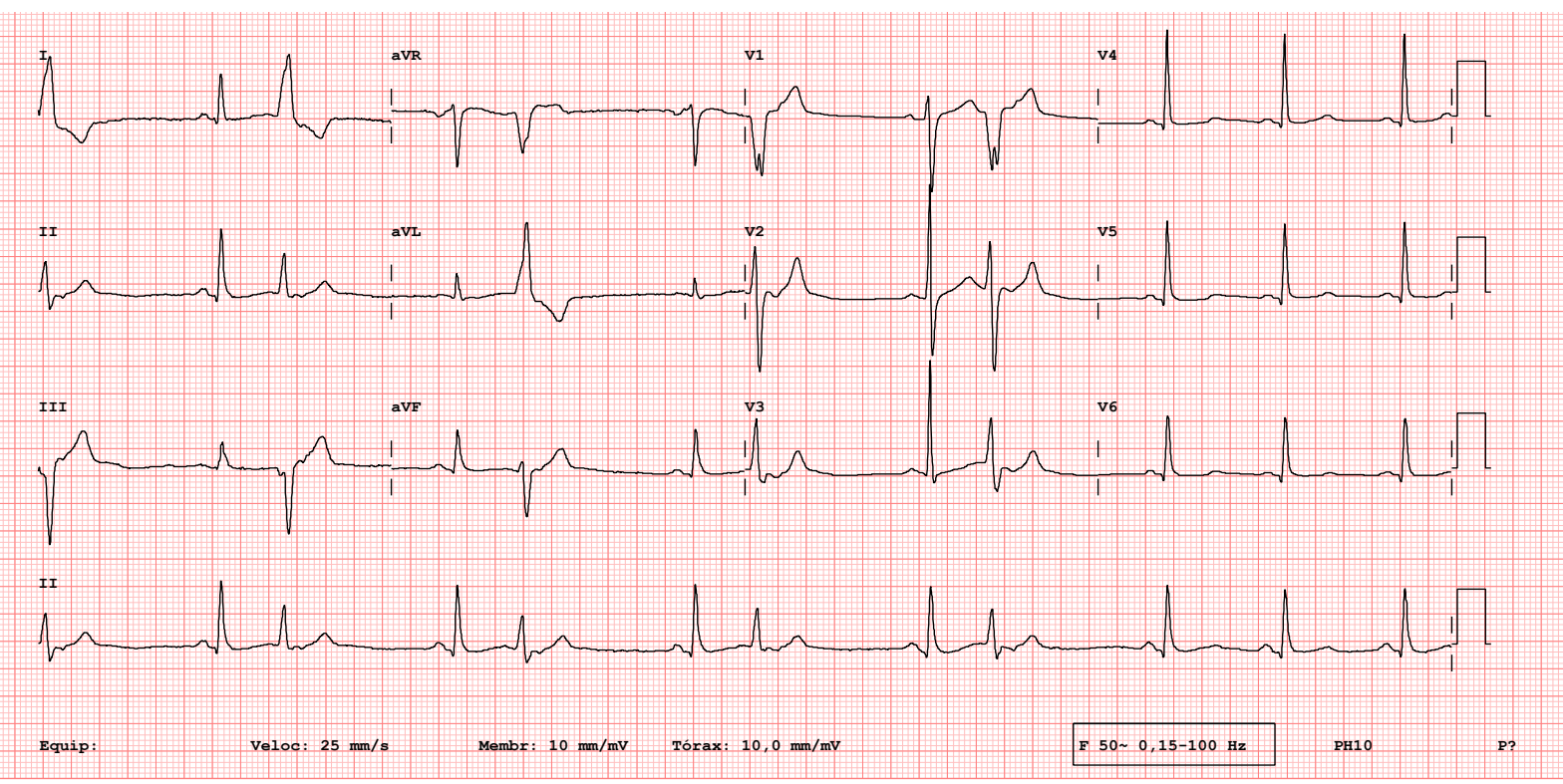

Figure 1 and 2: Pre and post-operative electrocardiography.

\section{Discussion:}

This clinical pattern was similar to cases of NE overdose previously described.[1] Despite uncommon, it highlights the importance of independent double-checking and strict signing of all medications before administration. Early recognition and support treatment are crucial to avoid fatal outcomes.

\section{Learning points:}

Despite all the anesthesiology evolution in the past decades, it is vital to always assure the patient safety in order to diminish the occurrence of avoidable errors.

\section{References:}

[1] Ibbey AA, et al. Inadvertent overinfusion of norepinephrine using infusion pump loading dose. Int Crit Care Nurs 2015 Dec; 31(6): 375-9.

[2] Arfi AA, et al. Acute myocardial ischemia following accidental intravenous administration of epinephrine in high concentration. Ind Heart J 2005; 57: 261-64.

[3] Ahmed DB, et al. Intraoperative Ventricular Fibrillation Arrest Caused by Accidental Intravenous Injection of Epinephrine. Ibn J Med BS 2014; 6(5): 223-226.

Corresponding Author: C. Monteiro Departament of Anesthesiology, Centro Hospitalar Universitário do Algarve - Unidade de Faro Rua Leão Penedo 8000-386 Faro (Portugal) E-mail: catarina_tm@hotmail.com 